Figure S2, panel 1. GBA N370S: G vs. A

\begin{tabular}{|c|c|c|}
\hline All studies & $\begin{array}{r}\text { OR } \\
3.45\end{array}$ & $\begin{array}{r}95 \% \mathrm{Cl} \\
{[2.70,4.40]}\end{array}$ \\
\hline All excl initial & 3.42 & {$[2.67,4.38]$} \\
\hline Caucasian studies & 3.51 & {$[2.55,4.83]$} \\
\hline Caucasian excl initial & 3.46 & {$[2.50,4.79]$} \\
\hline \multicolumn{3}{|l|}{ Study specific ORs } \\
\hline Do, $2011[\mathrm{C}]$ & 4.05 & {$[3.08,5.32]$} \\
\hline Lesage, $2011[\mathrm{O}]$ & 3.67 & {$[0.17,81.74]$} \\
\hline Lesage, $2010[\mathrm{C}]$ & 6.49 & {$[1.56,27.00]$} \\
\hline Santos, 2010 [O] & 7.76 & {$[0.39,155.65]$} \\
\hline $\mathrm{Hu}, 2010[\mathrm{~A}]$ & 2.76 & {$[0.55,13.73]$} \\
\hline Sun, $2010[\mathrm{~A}] \mathrm{i}$ & - & - \\
\hline Nishioka, 2009 [O] & 0.52 & {$[0.05,4.97]$} \\
\hline Sidransky, 2009, Japan [A] i & - & - \\
\hline Sidransky, 2009, Singapore $[\mathrm{A}]$ i & - & - \\
\hline Sidransky, 2009, Taiwan $[\mathrm{A}] \mathrm{i}$ & - & - \\
\hline Sidransky, 2009, France [C] & 6.84 & {$[0.85,54.87]$} \\
\hline Sidransky, 2009, Germany (Rostock) [C] & 3.58 & {$[0.42,30.74]$} \\
\hline Sidransky, 2009, Germany (Tuebingen) [C] & 12.19 & {$[0.69,215.25]$} \\
\hline Sidransky, 2009, Italy $[C] \ddagger$ & - & - \\
\hline Sidransky, 2009, Canada $[C] \ddagger$ & - & - \\
\hline Sidransky, 2009, Norway [C] $\ddagger$ & - & - \\
\hline Sidransky, 2009, Portugal [C] & 3.50 & {$[0.83,14.73]$} \\
\hline Sidransky, 2009, Brazil [O] & 8.19 & {$[0.27,245.59]$} \\
\hline Sidransky, 2009, USA [O] ‡ & - & - \\
\hline Sidransky, 2009, Israel [O] $\ddagger$ & - & - \\
\hline Sidransky, 2009, USA (NHGRI) [O] & 3.53 & {$[0.82,15.29]$} \\
\hline Sidransky, 2009, USA (Seattle) [O] & 4.50 & {$[1.02,19.85]$} \\
\hline Neumann, $2009[\mathrm{C}]$ & 2.61 & {$[0.33,20.92]$} \\
\hline Nichols, 2008 [C] & 1.60 & {$[0.40,6.42]$} \\
\hline Gan-Or, $2008[\mathrm{C}]$ & 2.31 & {$[1.70,3.13]$} \\
\hline Mata, $2008[\mathrm{C}] \ddagger$ & - & - \\
\hline De Marco, 2008 [C] & 7.37 & {$[0.37,147.32]$} \\
\hline Bras, $2007[\mathrm{C}] \ddagger$ & - & - \\
\hline Clark, 2007 [C] & 5.57 & {$[1.67,18.59]$} \\
\hline Toft, $2006[\mathrm{C}]$ & 0.87 & {$[0.25,2.98]$} \\
\hline Eblan, 2006 [O] & 1.80 & {$[0.06,54.65]$} \\
\hline Sato, 2005 [C] & 1.39 & {$[0.09,22.35]$} \\
\hline Clark, $2005[\mathrm{C}] \mp$ & - & - \\
\hline Aharon-Peretz, 2004 [C] & 5.58 & {$[3.58,8.72]$} \\
\hline Lwin, $2004[\mathrm{C}]$ & 11.80 & {$[0.66,210.85]$} \\
\hline
\end{tabular}

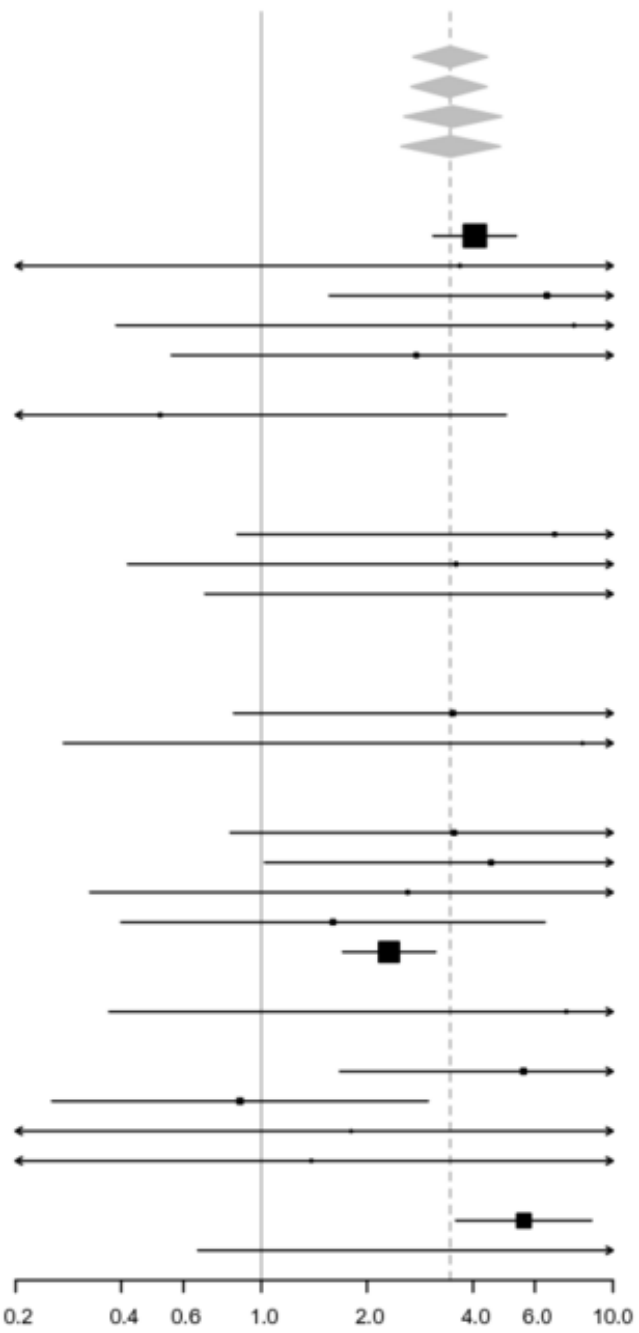


Figure S2, panel 2. SYT11/RAB25 chr1:154105678: T vs. C

\section{All studies}

Study specific ORs

Simon-Sanchez, $2011[\mathrm{C}]$

Saad, 2010 [C]

Spencer, 2010 [C]

Simon-Sanchez, 2009, Germany, GWAS [C]

Simon-Sanchez, 2009, USA-NINDS, GWAS [C]

Pankratz, 2008 [C]

\section{OR $\left.\quad 95 \% \mathrm{Cl}\right|^{2}$ $1.73[1.48,2.01] 0$}

$1.97[1.41,2.75]$

$1.96[1.20,3.19]$

$1.50[1.16,1.94]$

$1.97[1.21,3.20]$

$1.57[1.04,2.37]$

$1.91[1.17,3.12]$

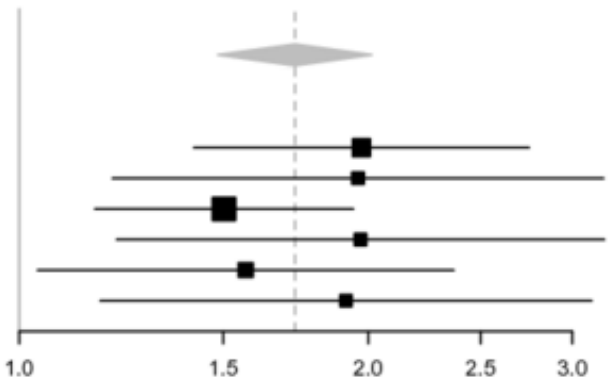

Figure S2, panel 3. PARK16 rs947211: A vs. G

All studies

Caucasian studies

Asian studies

Study specific ORs

Mata, 2011 [C]

Do, 2011 [C]

IPDGC, 2011 [C]

Simon-Sanchez, $2011[\mathrm{C}]$

Saad, $2010[\mathrm{C}]$

Spencer, $2010[\mathrm{C}]$

Ramirez, $2010[\mathrm{H}]$

Hamza, 2010 [C]

Tan, $2010[\mathrm{~A}]$

Edwards, $2010[\mathrm{C}]$

Satake, 2009, Japan, Replication-1 [A]

Satake, 2009, Japan, Replication-2 [A]

Satake, 2009, Japan, GWAS [A]

Simon-Sanchez, 2009, USA/Germany/UK, Stage II [C]

Simon-Sanchez, 2009, Germany, GWAS [C]

Simon-Sanchez, 2009, USA-NINDS, GWAS [C]

Pankratz, 2008 [C]

Fung, $2006[C] \ddagger$ $\begin{array}{rrr}\text { OR } & 95 \% \mathrm{Cl} & 1^{2} \\ 0.88 & {[0.85,0.91]} & 39 \\ 0.91 & {[0.88,0.94]} & 0 \\ 0.79 & {[0.73,0.85]} & 29\end{array}$

$0.95[0.83,1.09]$

$0.87[0.83,0.93]$

$0.91[0.77,1.08]$

$0.89[0.77,1.02]$

$0.85[0.75,0.97]$

$0.95[0.86,1.04]$

$0.84[0.63,1.13]$

$0.90[0.80,1.00]$

$0.88[0.75,1.04]$

$0.94[0.77,1.14]$

$0.74[0.66,0.83]$

$0.73[0.62,0.87]$

$0.82[0.74,0.90]$

$0.96[0.88,1.04]$

$0.84[0.71,1.00]$

$0.90[0.80,1.02]$

$0.97[0.83,1.14]$

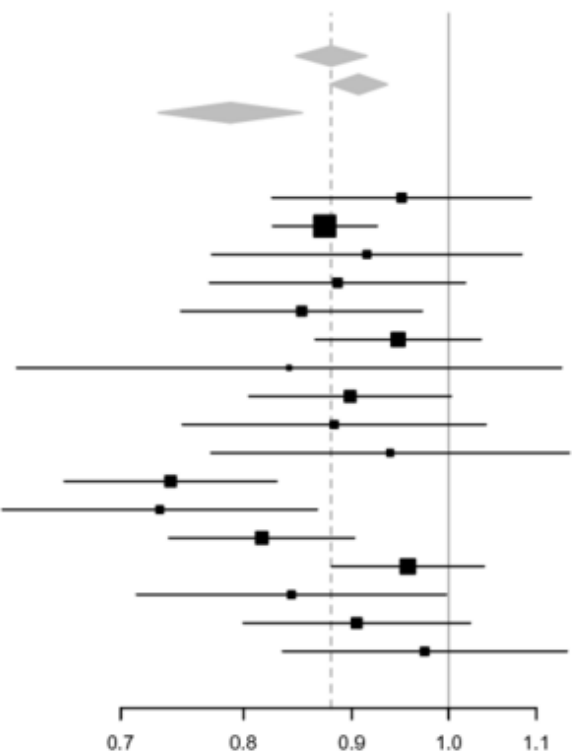

Figure S2, panel 4. STK39 rs2390669: C vs. A

All studies

Study specific ORs

IPDGC, 2011, France, REP [C]

IPDGC, 2011, Germany, REP [C]

IPDGC, 2011, Netherlands, REP [C]

IPDGC, 2011, UK, REP [C]

IPDGC, 2011, USA, REP [C]

IPDGC, 2011, Iceland, GWAS [C]

Simon-Sanchez, 2011 [C]

Saad, $2010[\mathrm{C}]$

Spencer, $2010[\mathrm{C}]$

Hamza, $2010[\mathrm{C}]$

Edwards, 2010 [C]

Simon-Sanchez, 2009, Germany, GWAS [C]

Simon-Sanchez, 2009, USA-NINDS, GWAS [C]

Pankratz, 2008 [C]
OR $\left.\quad 95 \% \mathrm{Cl} \quad\right|^{2}$

$1.19[1.12,1.25] 18$

1.07 [0.77,1.49]

$1.11[0.91,1.36]$

$1.35[0.99,1.82]$

$1.18[1.02,1.36]$

$1.12[1.00,1.25]$

$0.79[0.59,1.06]$

$1.11[0.90,1.35]$

$1.40 \quad[1.17,1.68]$

$1.22[1.07,1.39]$

$1.19[1.04,1.36]$

1.22 [0.93,1.59]

$1.36[1.05,1.76]$

$1.19[0.99,1.42]$

$1.35[1.07,1.71]$

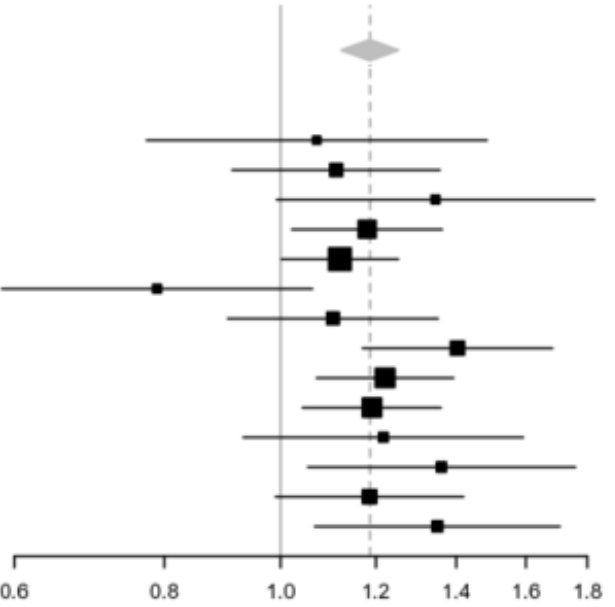


Figure S2, panel 5. MCCC1/LAMP3 rs11711441: A vs. G

\section{All studies \\ Caucasian studies \\ Asian studies}

Study specific ORs

GEO-PD, 2011, China-Mok [A]

GEO-PD, 2011, Korea-Jeon [A]

GEO-PD, 2011, Singapore-Tan [A]

GEO-PD, 2011, Taiwan-Lin [A]

GEO-PD, 2011, Australia-Mellick [C]

GEO-PD, 2011, Belgium-Garraux [C]

GEO-PD, 2011, Belgium-Van Broeckhoven [C]

GEO-PD, 2011, Greece-Bozi [C]

GEO-PD, 2011, Greece-Hadjigeorgios [C]

GEO-PD, 2011, Ireland-Lynch [C]

GEO-PD, 2011, Italy, Annesi [C]

GEO-PD, 2011, Norway-Aasley [C]

GEO-PD, 2011, France-Brice [C] $\ddagger$

GEO-PD, 2011, UK-Morrison [C] $\ddagger$

GEO-PD, 2011, Poland-Opala [C]

GEO-PD, 2011, Sweden-Wirdefeldt [C]

GEO-PD, 2011, USA-Farrer/Wszolek [C]

GEO-PD, 2011, USA-Maraganore [C]

IPDGC, 2011, France, REP [C]

IPDGC, 2011, Germany, REP [C]

IPDGC, 2011, Netherlands, REP [C]

IPDGC, 2011, UK, REP [C]

IPDGC, 2011, USA, REP [C]

IPDGC, 2011, Iceland, GWAS [C]

Simon-Sanchez, 2011 [C]

Saad, 2010 [C]

Spencer, $2010[\mathrm{C}]$

Hamza, 2010 [C]

Simon-Sanchez, 2009, Germany, GWAS [C]

Simon-Sanchez, 2009, USA-NINDS, GWAS [C]

Pankratz, 2008 [C]

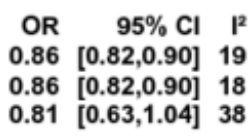

$0.68[0.47,0.99]$

$0.82[0.63,1.06]$

$1.35[0.78,2.32$

$0.66[0.40,1.09]$

$0.89[0.72,1.09]$

$1.61[0.46,5.58]$

$1.00[0.76,1.30]$

$1.33[0.77,2.29]$

$1.18 \quad[0.86,1.62]$

$0.56[0.39,0.80]$

$0.80[0.52,1.22]$

$0.76[0.59,0.97]$

- -

$0.86[0.58,1.28]$

$0.74[0.42,1.29]$

$0.97[0.76,1.22]$

$1.15[0.82,1.62]$

$0.69[0.48,0.99]$

$0.75[0.62,0.91]$

$0.90[0.67,1.19]$

$0.83[0.71,0.96]$

$0.92[0.82,1.03]$

$0.92[0.73,1.18]$

$0.94[0.80,1.11]$

$0.82[0.70,0.97]$

$0.83[0.74,0.93]$

$0.91[0.81,1.02]$

$0.78[0.63,0.95]$

$0.84[0.72,0.98]$

$0.78[0.64,0.96]$
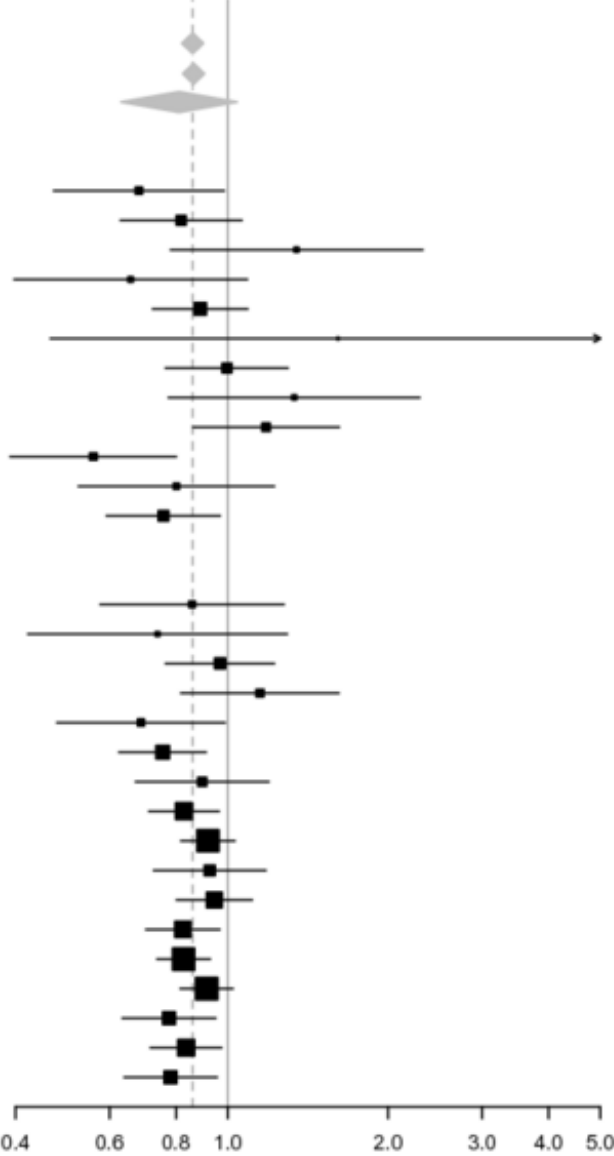

Figure S2, panel 6. DGKQ/GAK rs11248060: T vs. C

All studies

Study specific ORs

Do, $2011[\mathrm{C}]$

IPDGC, 2011 [C]

Simon-Sanchez, 2011 [C]

Saad, $2010[\mathrm{C}]$

Spencer, $2010[\mathrm{C}]$

Hamza, 2010 [C]

Edwards, 2010 [C]

Simon-Sanchez, 2009, Germany, GWAS [C]

Simon-Sanchez, 2009, USA-NINDS, GWAS [C]

Pankratz, 2008 [C]

Fung, $2006[C] \ddagger$
OR $\quad 95 \% \mathrm{Cl} \quad \mathrm{I}^{2}$ $1.21[1.14,1.27] 11$
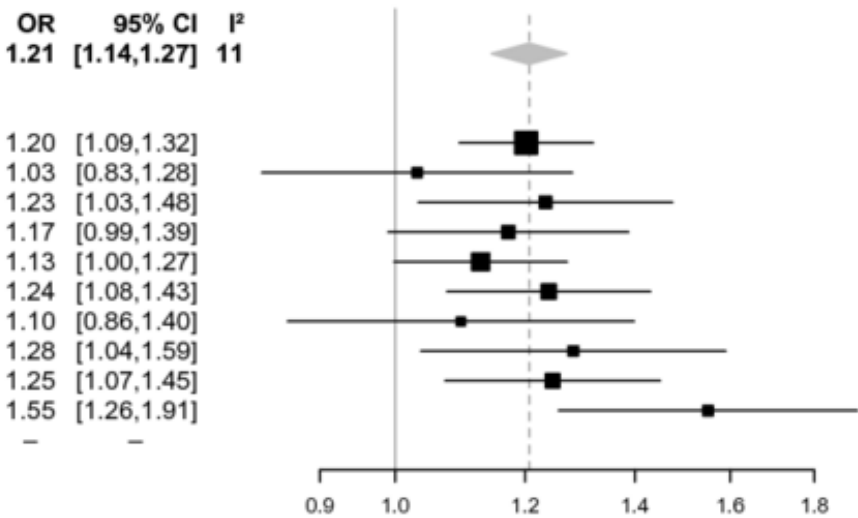
Figure S2, panel 7. BST1 rs11724635: C vs. A

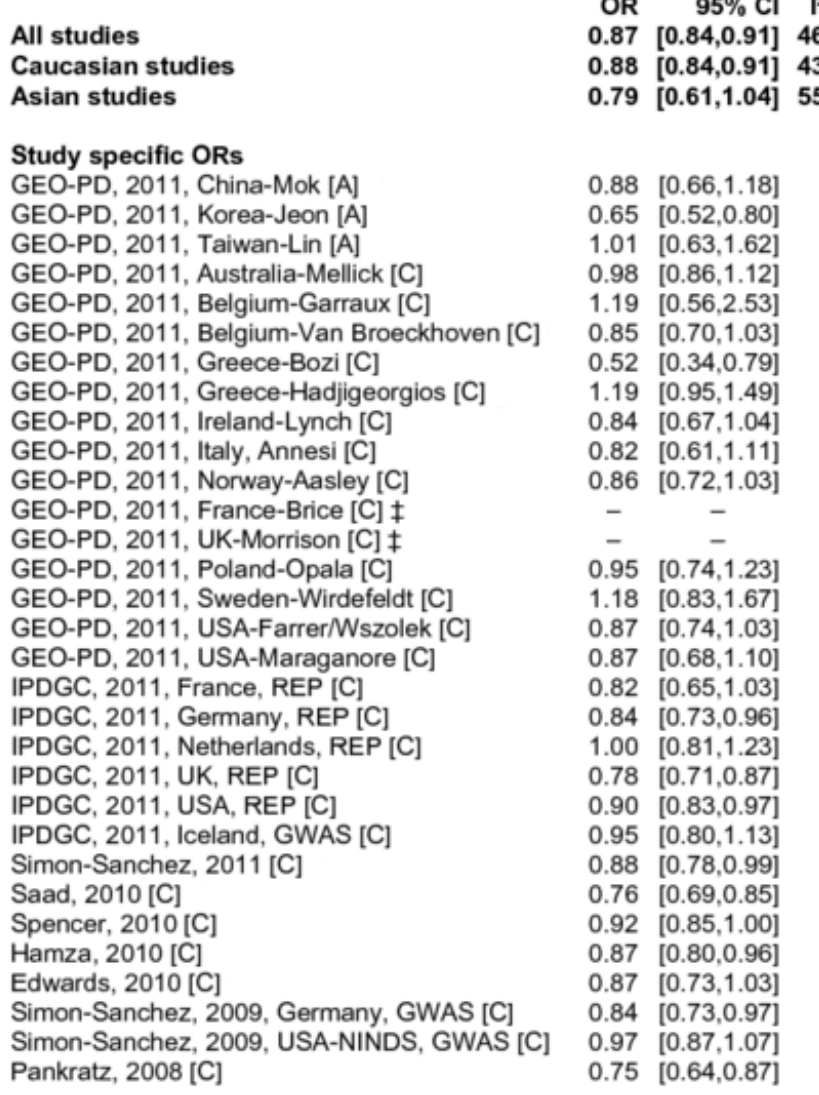

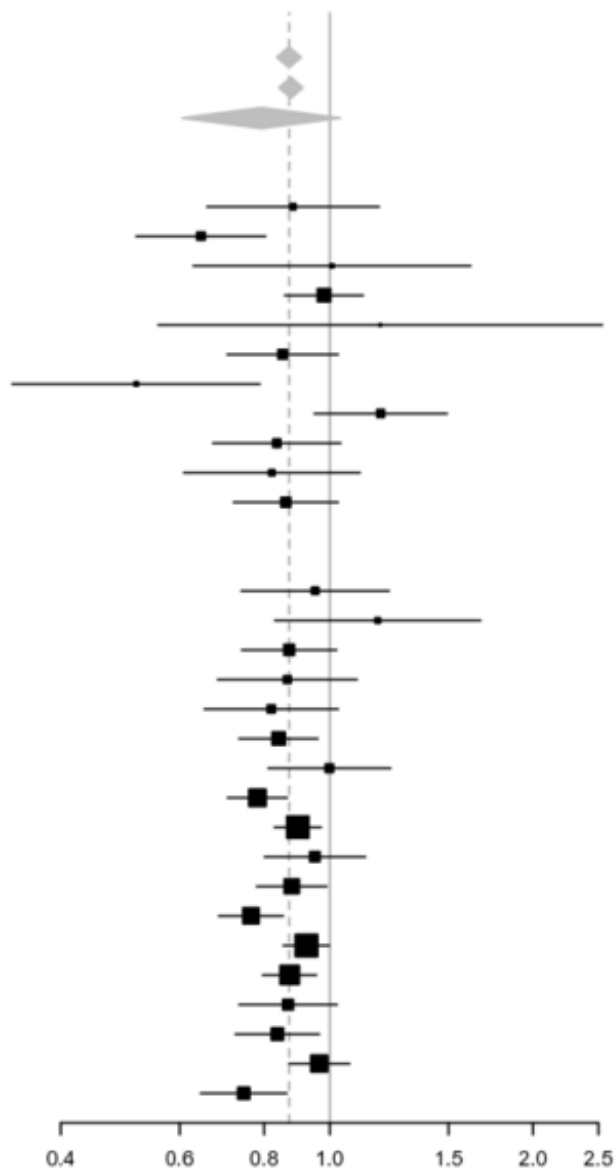


Figure S2, panel 8. SNCA rs356219: G vs. A

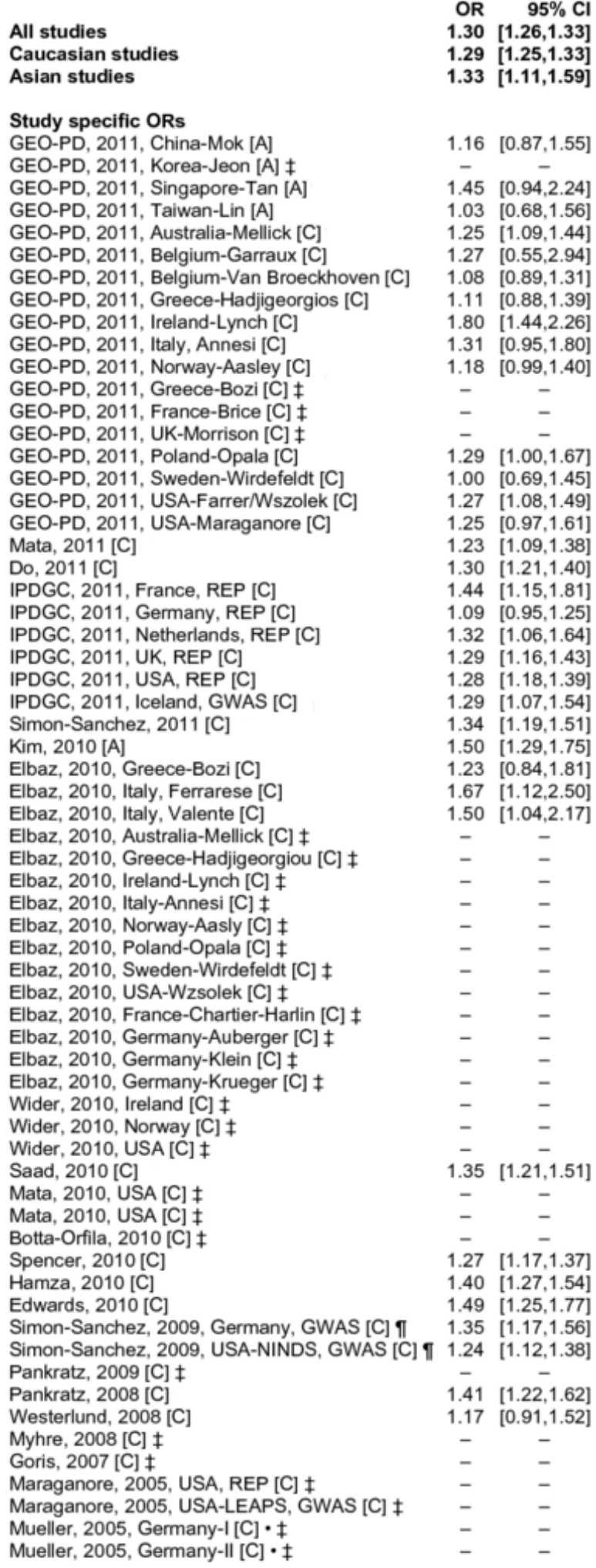

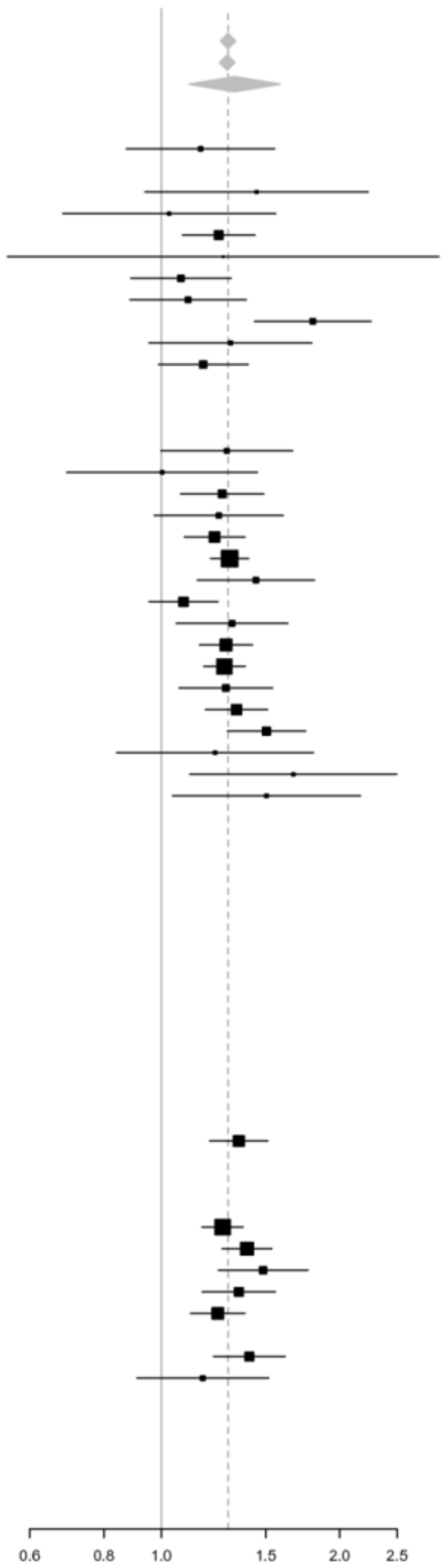


Figure S2, panel 9. ITGA8 rs7077361: C vs. T

$\begin{array}{lrr} & \text { OR } & \mathbf{9 5 \%} \mathbf{C l} \mathbf{I}^{2} \\ \text { All studies } & \mathbf{0 . 8 8} & {[\mathbf{0 . 8 4 , 0 . 9 2}]} \\ & & \\ \text { Study specific ORs } & & \\ \text { Do, 2011 [C] } & 0.90 & {[0.83,0.97]} \\ \text { IPDGC, 2011 [C] } & 0.79 & {[0.62,1.02]} \\ \text { Simon-Sanchez, 2011 [C] } & 0.80[0.67,0.97] \\ \text { Saad, 2010 [C] } & 0.88[0.74,1.04] \\ \text { Spencer, 2010 [C] } & 0.87 & {[0.77,0.98]} \\ \text { Hamza, 2010 [C] } & 0.97 & {[0.84,1.11]} \\ \text { Edwards, 2010 [C] } & 0.82 & {[0.63,1.06]} \\ \text { Simon-Sanchez, 2009, USA/Germany/UK, Stage II [C] } \ddagger & - & - \\ \text { Simon-Sanchez, 2009, Germany, GWAS [C] } & 0.71 & {[0.57,0.88]} \\ \text { Simon-Sanchez, 2009, USA-NINDS, GWAS [C] } & 0.83[0.71,0.98] \\ \text { Pankratz, 2008 [C] } & 0.92[0.76,1.13] \\ \text { Maraganore, 2005 [C] } & 0.98 & {[0.66,1.46]}\end{array}$

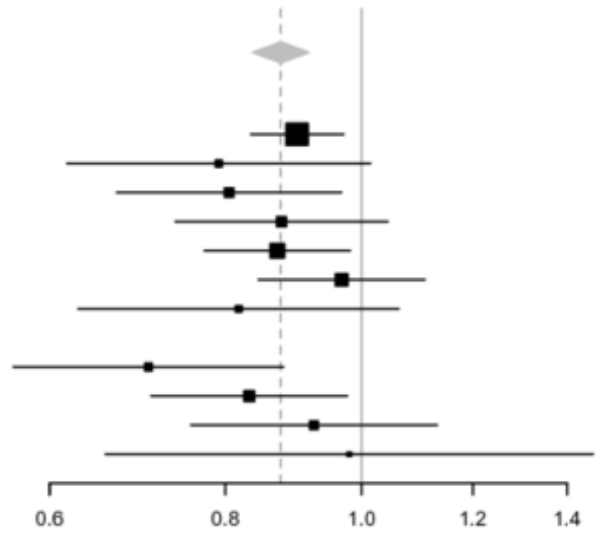

Figure S2, panel 10. LRRK2 rs1491942: G vs C

\begin{tabular}{|c|c|c|}
\hline & OR & $95 \% \mathrm{Cl}$ \\
\hline All studies & 1.17 & {$[1.13,1.22]$} \\
\hline Caucasian studies & 1.17 & {$[1.13,1.22]$} \\
\hline Asian studies & 1.12 & {$[0.96,1.31]$} \\
\hline \multicolumn{3}{|l|}{ Study specific ORs } \\
\hline GEO-PD, 2011, China-Mok [A] & 1.32 & {$[0.99,1.77]$} \\
\hline GEO-PD, 2011, Korea-Jeon [A] & 1.04 & {$[0.83,1.29]$} \\
\hline GEO-PD, 2011, Singapore-Tan [A] & 0.96 & {$[0.59,1.56]$} \\
\hline GEO-PD, 2011, Taiwan-Lin [A] & 1.23 & {$[0.78,1.93]$} \\
\hline GEO-PD, 2011, Australia-Mellick [C] & 1.08 & {$[0.91,1.28]$} \\
\hline GEO-PD, 2011, Belgium-Garraux [C] & 1.36 & {$[0.47,3.96]$} \\
\hline GEO-PD, 2011, Belgium-Van Broeckhoven [C] & 1.18 & {$[0.93,1.48]$} \\
\hline GEO-PD, 2011, Greece-Bozi [C] & 0.85 & {$[0.54,1.32]$} \\
\hline GEO-PD, 2011, Greece-Hadjigeorgios [C] & 1.08 & {$[0.83,1.40]$} \\
\hline GEO-PD, 2011, Ireland-Lynch [C] & 1.25 & {$[0.95,1.64]$} \\
\hline GEO-PD, 2011, Italy, Annesi [C] & 1.49 & {$[1.02,2.17]$} \\
\hline GEO-PD, 2011, Norway-Aasley [C] & 1.25 & {$[0.99,1.57]$} \\
\hline GEO-PD, 2011, France-Brice $[\mathrm{C}] \ddagger$ & - & - \\
\hline GEO-PD, 2011, UK-Morrison [C] $\ddagger$ & - & - \\
\hline GEO-PD, 2011, Poland-Opala [C] & 1.17 & {$[0.86,1.59]$} \\
\hline GEO-PD, 2011, Sweden-Wirdefeldt [C] & 1.25 & {$[0.79,1.96]$} \\
\hline GEO-PD, 2011, USA-Farrer/Wszolek [C] & 1.19 & {$[0.98,1.45]$} \\
\hline GEO-PD, 2011, USA-Maraganore [C] & 0.96 & {$[0.71,1.28]$} \\
\hline IPDGC, 2011 [C] & 1.05 & {$[0.85,1.30]$} \\
\hline Simon-Sanchez, $2011[\mathrm{C}]$ & 1.18 & {$[1.02,1.37]$} \\
\hline Saad, $2010[\mathrm{C}]$ & 1.26 & {$[1.10,1.45]$} \\
\hline Spencer, $2010[\mathrm{C}]$ & 1.11 & {$[1.01,1.22]$} \\
\hline Hamza, $2010[\mathrm{C}]$ & 1.25 & {$[1.12,1.40]$} \\
\hline Edwards, $2010[\mathrm{C}]$ & 1.07 & {$[0.87,1.31]$} \\
\hline Simon-Sanchez, 2009, Germany, GWAS [C] & 1.35 & {$[1.13,1.61]$} \\
\hline Simon-Sanchez, 2009, USA-NINDS, GWAS [C] & 1.24 & {$[1.09,1.42]$} \\
\hline Pankratz, 2008 [C] & 1.08 & {$[0.91,1.28]$} \\
\hline
\end{tabular}

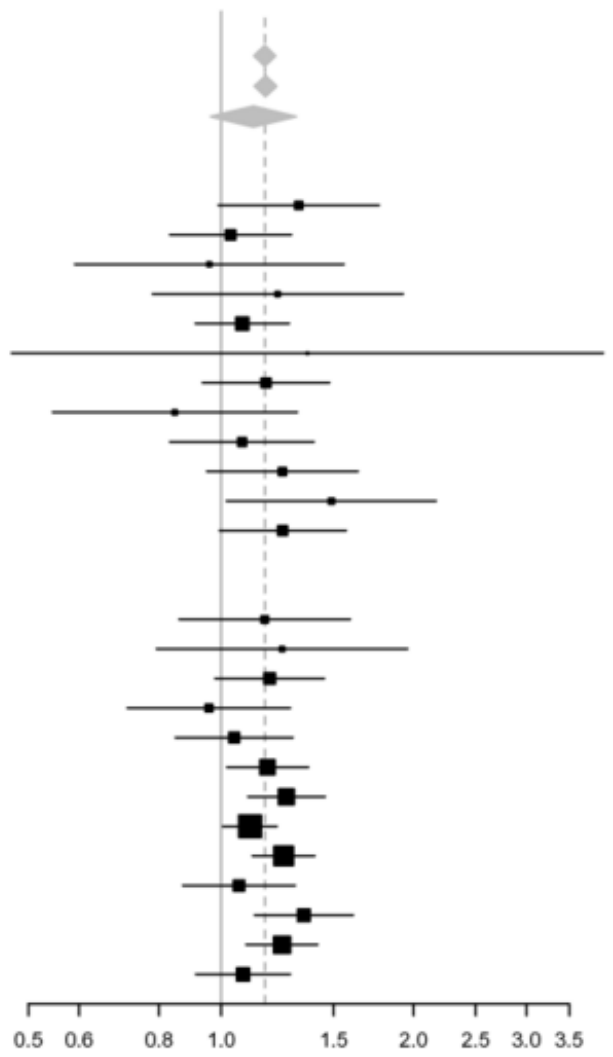


Figure S2, panel 11. CCDC62/HIP1R rs10847864: T vs G

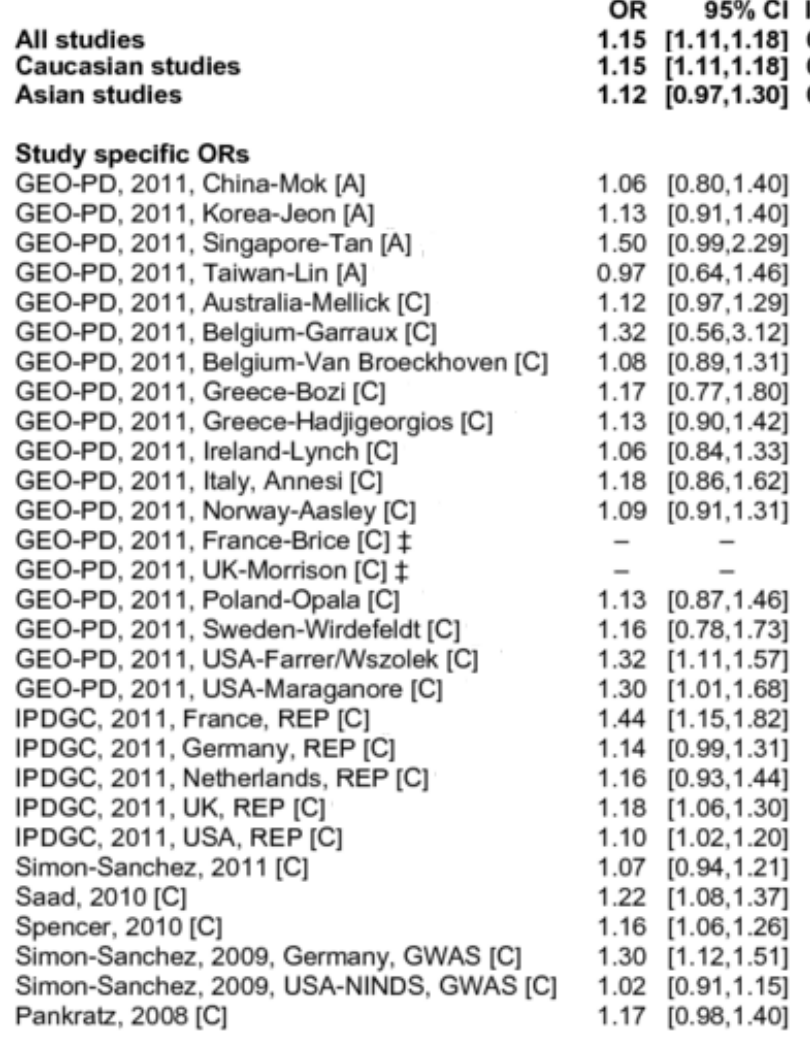

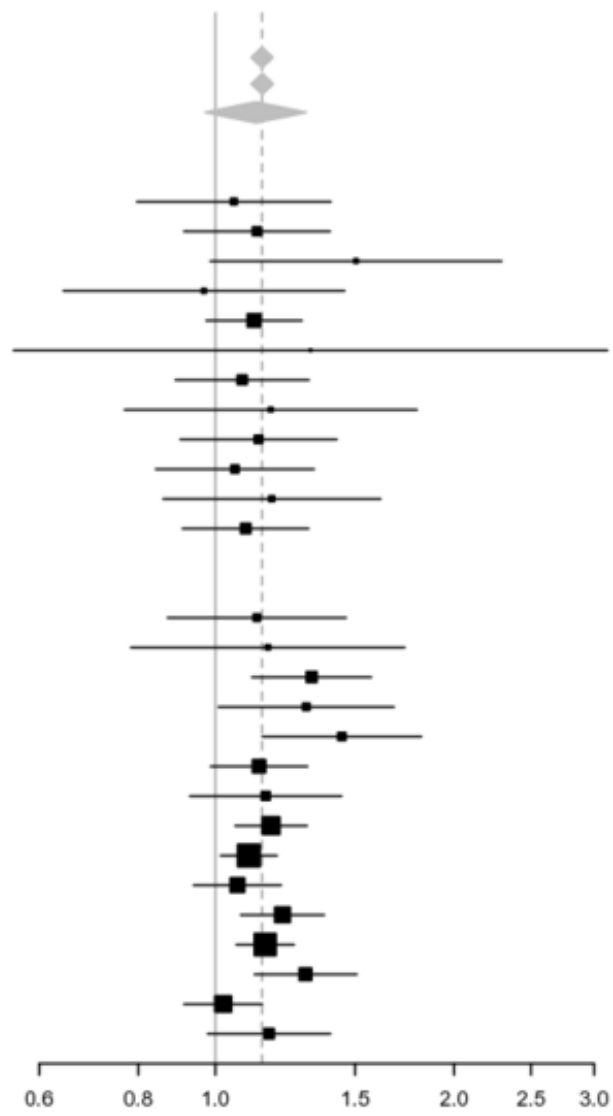


Figure S2, panel 12. MAPT H1H2: H2 vs H1

All studies ø

All excl HWE violations

Caucasian studies
Caucasian excl HWE violations

Study specific ORs

GEO-PD, 2011, China-Mok [A] i

GEO-PD, 2011, Singapore-Tan [A] i

GEO-PD, 2011, Taiwan-Lin [A]

GEO-PD, 2011, Australia-Mellick [C]
GEO-PD, 2011, Belgium-Garraux [C]

GEO-PD, 2011, Belgium-Garraux [C]

GEO-PD, 2011, Greece-Bozi [C]

GEO-PD, 2011, Greece-Hadjigeorgios [C]

GEO-PD, 2011, Italy-Annesi [C]

GEO-PD, 2011, Norway-Aasley [C]

GEO-PD, 2011, Ireland-Lynch [C] ‡

GEO-PD, 2011, Poland-Opala [C] $\ddagger$

GEO-PD, 2011, Sweden-Wirdefeldt

GEO-PD, 2011, France-Brice [C] $\ddagger$

GEO-PD, 2011, UK-Morrison [C] $\ddagger$

GEO-PD, 2011, USA-Maraganore [C]

Mata, 2011 [C]

IPDGC, 2011, France, REP [C]

IPDGC, 2011, Germany, REP [C]

IPDGC, 2011, Netherlands, REP [C]

IPDGC, 2011, UK, REP [C]

IPDGC, 2011, USA, REP [C]

Simon-Sanchez, 2011 [C]

Elbaz, 2010, Ireland (Lynch) [C]

Elbaz, 2010, Italy (Ferrarese) [C] \#

Elbaz, 2010, Italy (Valente) [C]

Elbaz, 2010, Australia-Mellick [C]

Elbaz, 2010, Greece-Bozi [C] $\ddagger$

Elbaz, 2010, Greece-Hadjigeorg

Elbaz, 2010, Italy-Annesi [C] $\ddagger$

Elbaz, 2010, Norway-Aasly $[C] \ddagger$

Elbaz, 2010, USA-Farrer $[C] \ddagger$
Elbaz, 2010, Germany/Auberger [C] $\ddagger$

Elbaz, 2010, France $[\mathrm{C}] \ddagger$

Elbaz, 2010, Germany/Klein [C] $\ddagger$

Elbaz, 2010, Germany/Krueger [C] $\ddagger$

Elbaz, 2010, Poland (Opala) [C] \#

Elbaz, 2010, Sweden (Wirdefeldt) [C]

Wider, 2010, Ireland [C]

Wider, 2010, Norway-Aasly [C] $\ddagger$

Wider, 2010, USA [C] :

Chung, $2010[\mathrm{C}]$

Botta-Orfila, $2010[\mathrm{C}] \mp$

Spencer, $2010[\mathrm{C}]$

Benitez, 2010 [O]

Hamza, $2010[\mathrm{C}]$

Edwards, $2010[\mathrm{C}]$

OR $\quad 95 \% \mathrm{Cl} \mathrm{l^{2 }}$

$0.78 \quad[0.75,0.80] 1$

$0.78[0.76,0.81] 0$

$0.78[0.75,0.80] 0$

IC

Wider, 2009, Ireland [C]

Wider, 2009, Norway-Aasly [C] $\ddagger$

Wider, 2009, USA [C] $\ddagger$

Ezquerra, $2009[\mathrm{C}] \ddagger$

Payami, $2009[\mathrm{C}] \dagger$

Kalinderi, $2009[\mathrm{C}] \#$

Refenes, $2009[\mathrm{C}] \#$

Garcia-Gorostiaga, $2009[\mathrm{C}]$

Garcia-Gorostiaga, 2009 [O]

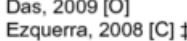

Ezquerra, $2008[\mathrm{C}] \ddagger$

McCulloch, 2008, USA-Georgia [C] $\ddagger$

McCulloch, 2008, USA-NY [C] ‡

McCulloch, 2008, USA-Oregon [C]

McCulloch, 2008, USA-Washington [C]

Vandrovcova, $2007[\mathrm{C}] \ddagger$

Goris, $2007[\mathrm{C}] \ddagger$

Winkler, 2007, Germany/Klein [C] $\ddagger$

Winkler, 2007, Serbia [C]

Zabetian, $2007[\mathrm{C}] \mp$
Papapetropoulos, $2007[\mathrm{C}]$

Fung, 2006, Taiwan [A]

Fung, 2006, Taiwan $[A]$

Fung, 2006, Finland $[C]$

Fung, 2006, Greece [C]

Kobayashi, 2006 [A]

Fidani, $2006[\mathrm{C}] \ddagger$

Johansson, $2005[\mathrm{C}]$

Kwok, 2005, China [A]

Kwok, 2005, Australia $[C] \ddagger$

Maraganore, $2005[\mathrm{C}] \neq$

Mamah, $2005[\mathrm{C}] \ddagger$

Skipper, $2004[\mathrm{C}] \ddagger$

Healy, $2004[\mathrm{C}] \ddagger$

Kwok, 2004 [C] $\ddagger$

Levecque, $2004[\mathrm{C}]$

Peplonska, 2003 [C]

Peplonska, 2003 [C]

Clark, 2003, USA [O]

de Silva, $2002[\mathrm{C}]$

Farrer, $2002[\mathrm{C}] \neq$

Maraganore, $2001[\mathrm{C}] \cdot \ddagger$

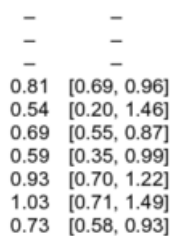

$0.73[0.58,0.93]$

$\begin{array}{ll}0 & - \\ - & -\end{array}$

- $\quad-$

$\begin{array}{ll}- & - \\ - & -\end{array}$

$0.68 \quad[0.55,0.83]$

$0.76[0.57,1.03]$

$0.79[0.70,0.90]$

$0.86[0.66,1.11]$

$0.86[0.72,1.02]$

$0.92[0.71,1.19]$

$0.79[0.70,0.89]$

$0.76[0.69,0.83]$

$0.82[0.71,0.95]$

$0.67[0.52,0.87]$

$0.61[0.39,0.97]$

$0.91[0.61,1.36]$

- -

$\begin{array}{ll}- & - \\ - & -\end{array}$

-

-

-

$0.91 \quad[0.67,1.24]$

$1.06[0.64,1.75]$

$\begin{array}{ll}- & - \\ - & -\end{array}$

- -

$0.84[0.66,1.06]$

$0.74[0.64,0.86]$

$\overline{0.77} \quad[0.70,0.84]$

$0.76[0.46,1.26]$

$0.76[0.68,0.86]$

$0.76[0.61,0.94]$

$0.71[0.59,0.85]$

$0.81[0.71,0.93]$

-

-

$59[0.42,0.84]$

$0.59[0.38,0.92]$

$\begin{array}{ll}0.59 & {[0.38,0.92]} \\ 0.95 & {[0.71,1.27]}\end{array}$

$0.95[0.71,1.27]$
$1.35 \quad[0.77,2.39]$

$1.35[0.77,2.39]$

$2.23[0.37,13.59]$

- $\quad-$

-

- $\quad-$

$.57[0.39,0.83]$

$\begin{array}{ll}- & - \\ 0 & {[0.34,0.89]}\end{array}$

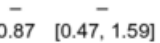

$\begin{array}{cc}0.87 & {[0.47,1.59} \\ - & -\end{array}$

$-\quad-$

$0.68[0.41,1.14]$

- $\quad-$

-

-

- -

- $92 .-\overline{-}[0.52,1.62]$

$0.82[0.52,1.62]$

\begin{tabular}{ll}
0.82 & {$[0.47,1.43]$} \\
\hline & {$[0.76,2.88]$}
\end{tabular}

- $\quad-$

$\begin{array}{ll}- & - \\ - & -\end{array}$

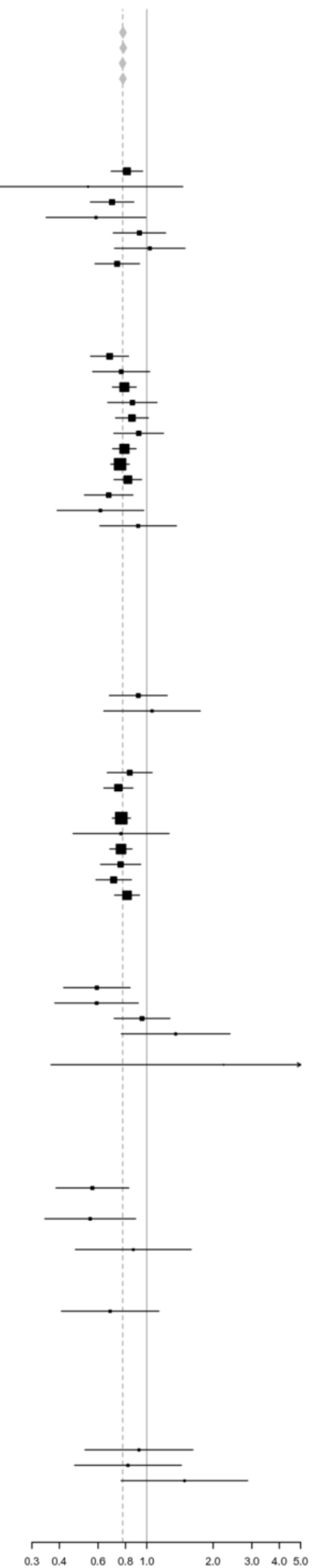


Figure S2, panel 13. PARK16 rs823156: G vs A

All studies

Caucasian studies

Asian studies

Study specific ORs

Mata, 2011 [C]

Do, 2011 [C]

IPDGC, 2011 [C]

Chang, 2011 [A]

Simon-Sanchez, 2011 [C]

Saad, $2010[\mathrm{C}]$

Spencer, $2010[\mathrm{C}]$

Hamza, 2010 [C]

Tan, $2010[\mathrm{~A}]$

Edwards, $2010[\mathrm{C}]$

Satake, 2009, Japan, Replication-1 [A]

Satake, 2009, Japan, Replication-2 [A]

Satake, 2009, Japan, GWAS [A]

Simon-Sanchez, 2009, USAVGermany/UK, Stage II [C] ‡

Simon-Sanchez, 2009, Germany, GWAS [C]

Simon-Sanchez, 2009, USA-NINDS, GWAS [C]

Pankratz, 2008 [C]

Fung, $2006[C] \ddagger$

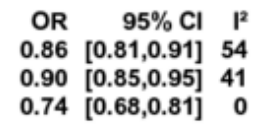

$0.88[0.75,1.03]$

$0.83[0.78,0.88]$

$0.94[0.66,1.35]$

$0.74[0.60,0.92]$

$0.99[0.84,1.16]$

$0.83[0.72,0.96]$

$0.98[0.88,1.08]$

$0.91[0.80,1.03]$

$0.77[0.63,0.95]$

$1.10[0.88,1.38]$

$0.80[0.68,0.94]$

$0.66[0.51,0.85]$

$0.72[0.62,0.83]$

\section{$-$}

$0.88[0.73,1.06]$

$0.83[0.72,0.95]$

$0.98[0.82,1.17]$

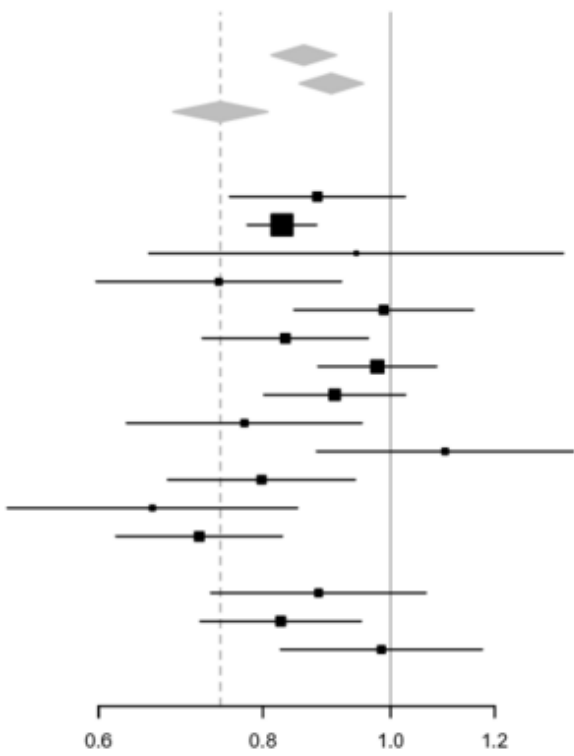

Figure S2, panel 14. BST1 rs4538475: G vs A

All studies

All excl initial

Caucasian studies

Caucasian excl initial

Asian studies

OR $\quad 95 \% \mathrm{Cl} \quad \mathrm{I}^{2}$

0.89 [0.84,0.93] 40

$0.88[0.84,0.92] 31$

$0.91[0.87,0.96] 21$

$0.91[0.87,0.94]$

$0.80[0.75,0.86]$

Study specific ORs

Do, 2011 [C]

IPDGC, 2011 [C]

Simon-Sanchez, 2011 [C]

Saad, 2010 [C]

Spencer, $2010[\mathrm{C}]$

Hamza, 2010 [C]

Edwards, 2010 [C]

Satake, 2009, Japan, Replication-1 [A]

Satake, 2009, Japan, Replication-2 [A]

Satake, 2009, Japan, GWAS [A]

Simon-Sanchez, 2009, USAVGermany/UK, Stage II [C] ‡

Simon-Sanchez, 2009, Germany, GWAS [C]

Simon-Sanchez, 2009, USA-NINDS, GWAS [C]

Pankratz, $2008[\mathrm{C}]$

Fung, $2006[\mathrm{C}] \ddagger$

Maraganore, $2005[\mathrm{C}]$

$0.89[0.83,0.96]$

$1.04[0.86,1.25]$

$0.96[0.83,1.13]$

$0.83[0.71,0.97]$

$0.87[0.78,0.97]$

$0.90[0.79,1.02]$

$0.88[0.69,1.11]$

$0.80[0.71,0.90]$

$0.82[0.69,0.97]$

$0.80[0.72,0.89]$

$-$

$0.87[0.71,1.05]$

$1.04[0.90,1.20]$

$0.84[0.70,1.02]$

$-$

$1.32[0.90,1.94]$

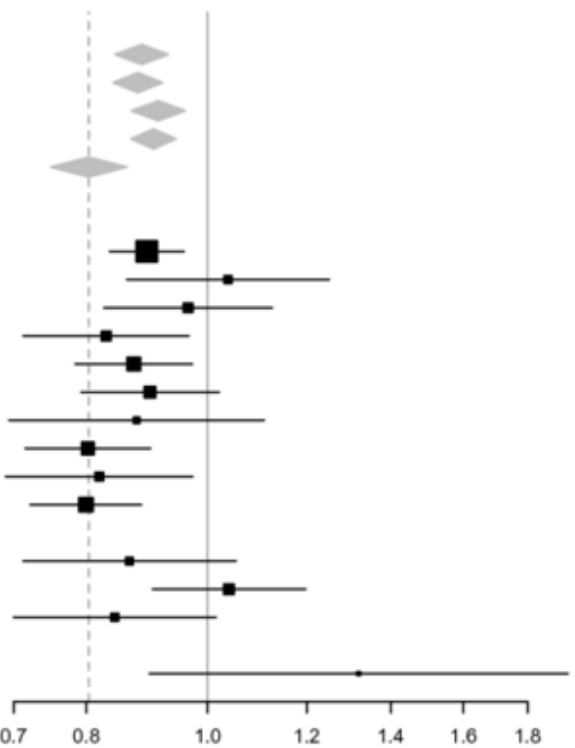

Figure S2, panel 15. SNCA rs6532194: T vs C

All studies

Asian studies

Caucasian studies

Study specific ORs

Chang, 2011 [A]

Simon-Sanchez, 2011 [C]

Saad, 2010 [C]

Tan, 2010 [A]

OR $\quad 95 \% \mathrm{Cl} \quad \mathrm{I}^{2}$

$1.23[1.14,1.32] 45$

$1.29[1.20,1.39] 31$

$1.14[1.02,1.27] 27$

Satake, 2009, Japan (Replication-2) [A] 1.21 [1.01,1.44]

Satake, 2009, Japan (Replication-1) [A] 1.22 [1.08,1.37]

Satake, 2009, Japan (GWAS) [A]

Simon-Sanchez, 2009 [C]

Pankratz, 2008 [C]

Fung, $2006[\mathrm{C}] \ddagger$
$1.27[1.07,1.50]$

$1.22[1.02,1.46]$

$1.26[1.07,1.48]$

$1.44[1.30,1.61]$

$1.23[1.06,1.42]$

$0.95[0.75,1.20]$
$1.08[0.87,1.34]$

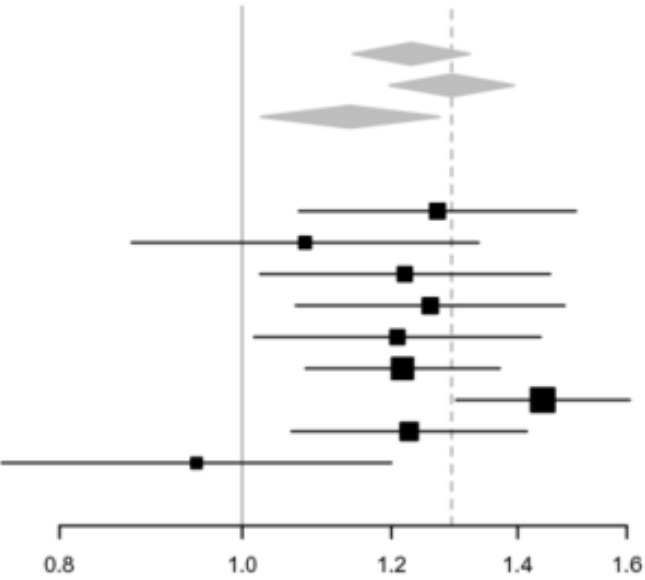


Figure S2, panel 16. LRKK2 rs34778348: A vs G

All studies

All excl initial

Asian studies

Asian excl initial

Study specific ORs

Lin, 2010 [A]

Miyake, 2010 [A]

Pulkes, $2010[\mathrm{~A}]$

Tan, 2010, Singapore II [A] $\ddagger$

Tan, 2010, Singapore I [A] $\ddagger$

Tan, 2010, Taiwan I [A] $\ddagger$

Tan, 2010, China [A]

Kim, 2009 [A]

Nuytemans, 2009 [C]

Zabetian, 2009 [A]

Choi, 2008 [A]

Chan, 2008 [A]

An, $2008[\mathrm{~A}] \ddagger$

Li, 2007 [A]

Tan, 2007, Singapore (Indian) [O]

Tan, 2007, Singapore (Malay) [O]

Funayama, 2007 [A]

Farrer, $2007[\mathrm{~A}] \ddagger$

Fung, 2006 [A]

Tan, 2006 [A]

Di Fonzo, 2006 [A] -

$\begin{array}{rrr}\text { OR } & 95 \% \mathrm{Cl} & \mathrm{l}^{2} \\ 2.11 & {[1.61,2.75]} & 58 \\ 2.12 & {[1.58,2.84]} & 61 \\ 2.23 & {[1.89,2.63]} & 0 \\ 2.24 & {[1.88,2.67]} & 1\end{array}$

$1.69[0.89,3.24]$

$2.11[1.21,3.68]$

$2.04[0.07,60.91]$

$\begin{array}{ll}- & - \\ - & -\end{array}$

- $\quad-$

$3.18[1.93,5.24]$

$1.87[1.15,3.04]$

$0.94[0.68,1.29]$

$1.96[1.44,2.68]$

$2.60[0.85,7.93]$

$3.23[0.40,26.37]$

$-$

$13.17[1.72,100.59]$

$\overline{1.76}[0.25,-12.57]$

$2.60[1.57,4.31]$

$-$

$16.26[2.20,120.16]$

$2.15[1.22,3.80]$

$2.14[1.25,3.64]$

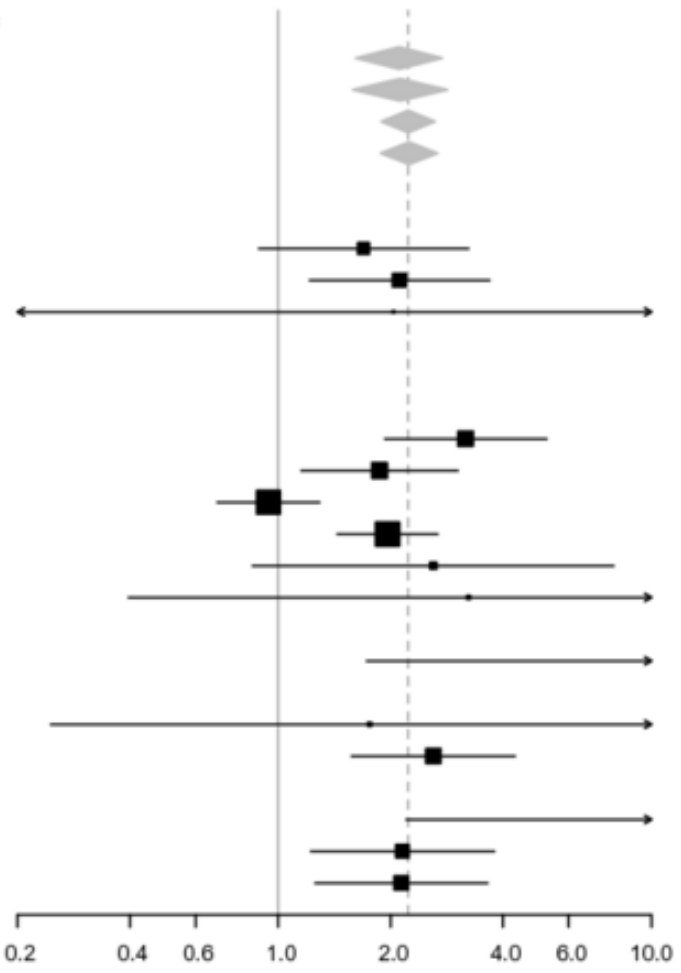

